\title{
Conception and Evaluation of a Biopolymer Cream Extracted from a Marine Biomass-Anti-Infectious Effect and Healing of Chronic Wounds
}

\author{
Laribi-Habchi Hassiba ${ }^{1,2, *}$, Saadoune Zineb ${ }^{1}$, Amrouche Zoheir ${ }^{3}$ \\ ${ }^{1}$ Laboratory of Functional Analysis of Chemical Processes (LFACP), Process Engineering Department, Faculty of Technology, University \\ of Blida, Blida, Blida Province, Algeria. \\ ${ }^{2}$ Loboratory of Energy Applications of Hydrogen, University Saad Dahlab of Blida 1, P.O. Box 270, 09000, Blida, Blida Province, Alge- \\ ria. \\ ${ }^{3}$ Biological Department, Faculty of Science of the Nature and the Life, University of Djilali Bounaam, Khemis Miliana, Algeria.
}

\begin{abstract}
How to cite this paper: Laribi-Habchi Hassiba, Saadoune Zineb, Amrouche Zoheir. (2021). Conception and Evaluation of a Biopolymer Cream Extracted from a Marine Biomass-Anti-Infectious Effect and Healing of Chronic Wounds. Advance in Biological Research, 2(1), 1-7.

DOI: 10.26855/abr.2021.11.001
\end{abstract}

Received: September 12, 2021

Accepted: October 8, 2021

Published: November 4, 2021

Corresponding author: Laribi-Habchi Hassiba, Laboratory of Functional Analysis of Chemical Processes (LFACP), Process Engineering Department, Faculty of Technology, University of Blida, Blida, Blida Province, Algeria; Loboratory of Energy Applications of Hydrogen, University Saad Dahlab of Blida 1, P.O. Box 270, 09000, Blida, Blida Province, Algeria.

Email: larhassiba@yahoo.fr

\begin{abstract}
Antibiotic resistance is a threat to global health. This growing microbial resistance could kill millions of people. Chronic wounds prone to colonization by infectious pathogens that are difficult to treat because they develop in biofilms. In this context, an organic galenic formulation has enabled the development of an anti-infectious and healing cream based on an active principle, Beta chitosan with a degree of deacetylation of $70 \%$ and a molecular weight of $65 \mathrm{KDa}$ extracted. Beta chitin from the bone of Sepia officinalis from the Mediterranean Sea in Algeria using shea butter, sweet almond oil and beeswax as excipients. The studied parameters of the cream, the antimicrobial evaluations, the rheological tests carried out as well as the preclinical tests carried out on animals with deep wounds caused by burns produced satisfactory results after 7 days of treatment compared to a cream conventional (21 days). Proven effectiveness can be sold at a lower cost compared to healing and antimicrobials.
\end{abstract}

\section{Keywords}

Beta chitosan, Shea butter, Healing cream, Sepia officinalis

\section{Introduction}

Chitosan is a linear polysaccharide derived from the partial deacetylation of chitin $[1,2,3]$. Its chemical structure, similar to that of cellulose, is composed of a random arrangement of D-glucosamine and Nacetyl-D-glucosamine units linked together by $\beta$-(1-4) bonds [4]. Having three functional groups, an amine group and two hydroxyl groups, the cationic nature of chitosan gives it a particular property compared to the majority of polysaccharides which are generally either neutral or negatively charged in an acidic medium [1]. The biocompatibility, biodegradability, low allergenicity and non-toxicity of chitosan are all characteristics that allow it to be used in a variety of applications [5, 6].

Chitosan is known primarily for its chelating properties. However, it has good antimicrobial properties against different groups of microorganisms: bacteria, viruses, fungi, yeasts and moulds [7, 8]. In general, moulds and yeasts are the most sensitive to chitosan followed by bacteria. The antimicrobial activity of chitosan is influenced by many factors, including the biological origin of chitosan, percentage deacetylation, degree of polymerization or molecular weight, and the type of 
target organisms. The response of the microorganism depends on the chemical structure of the chitosan used and the environmental conditions during the interaction [10].

Chronic wounds represent a real public problem, the prevalence of which increases with age. The infection of chronic wounds (burns or others) is serious with the delay of the cicatrization from where repeated care is necessary to fight the infection. Otherwise, bacterial proliferation can lead to the formation of a bio film favoring bacterial protection against the body's natural defenses and broad spectrum antimicrobial agents. Today, existing and available on the markets pharmaceutical and para pharmaceutical products based on synthetic raw materials harmful to human health and also on the environment. On this basis, the search for bio products has become a major concern in the field of scientific research.

In this perspective, the objective of our study is to evaluate the biological interest of a bio-therapeutic cream based on an active principle which is the beta chitosan (derived from chitin) prepared from the bone of dry Sepia officinalis of the Mediterranean Sea of Algeria. An in vivo application this formulation has been realized as anti-burn and healing of deep infected wounds.

\section{Material and Methods}

\subsection{Sample preparation}

Fresh bones of Sepia were washed thoroughly with tap water and they were air dried overnight. The Sepia bones were crushed into smaller pieces. The samples were oven-dried for 20 hat $100^{\circ} \mathrm{C}$.

\subsection{Extraction of chitin and chitosan}

\subsubsection{Demineralization}

Crushed Sepia bones were demineralized using with1.5M HCl. The samples were allowed to soak for one hour to remove the minerals (mainly calcium carbonate). The demineralized shrimp shell samples were then washed with deionized water, which is then drained off.

\subsubsection{Deproteinization}

The grounded Sepia bones were placed in a beaker and soaked in boiling sodium hydroxide (1.25M) for 18hour in order to dissolve the proteins and sugars thus isolating the crude chitin. After the samples are boiled in the sodium hydroxide, the beakers containing the Sepia bones samples are removed from the hotplate, and allowed to cool for 30 minutes at room temperature. The remaining chitin is washed with deionized water, which is then drained off.

\subsubsection{Deacetylation}

For deacetylation, chitin was treated with strong alkali, $50 \% \mathrm{NaOH}$ was added and then boiled at $100^{\circ} \mathrm{C}$ for 24 hour. Followed by washing till it reaches neutral $\mathrm{pH}$. After drying at $50^{\circ} \mathrm{C}$ for 24 hour, the final product recovered was chitosan.

\subsection{Characterization of prepared chitosan}

\subsubsection{Molecular weight}

Molecular weight of chitosan sample was estimated by determination of its intrinsic viscosity using Brookfield viscometer. $1 \%$ chitosan solution (w/v) was prepared using acetic acid $1 \%$. The average molecular weight was calculated from Mark-Houwink equation:

$$
\eta=K M^{a}
$$

Where $[\eta]$ is the intrinsic viscosity, $\mathrm{M}$ is average molecular weight of the solution respectively and $\mathrm{K}$ and a are the Mark-Houwink constants specific for a given polymer [11].

\subsubsection{Degree of deacetylation}

The degree of deacetylation was determined using an infrared spectrometer. An aliquot of chitosan sample was compressed into pellets. The absorbances at 1,655 and $3,450 \mathrm{~cm}^{-}{ }^{1}$ were used to calculate the DD according to the formula of [12].

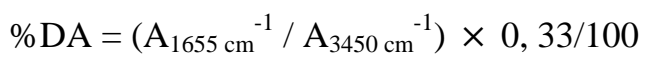

\subsubsection{Antimicrobial activity}

Antibacterial activities of chitosan were tested against four strains of bacteria; Gram-negative: Pseudomonas aeruginosa (ATCC 27853); and Gram-positive: Staphylococcus aureus (ATCC 25923), Bacillus cereus (ATCC 11778). Antifungal 
activities were tested against Aspergillus niger and Candida albicans. Chitosan solution (0.75\%) was prepared under stirring in acetic acid. Primazol and Lamidaz were used as positive references for bacteria and fungi activities, respectively. The Petri dishes were kept, first for $1 \mathrm{~h}$ at $4^{\circ} \mathrm{C}$, and then incubated for $24 \mathrm{~h}$ at $37^{\circ} \mathrm{C}$ for bacteria and $72 \mathrm{~h}$ at $30^{\circ} \mathrm{C}$ for fungal strains. Antimicrobial activity was evaluated by measuring the diameter of growth inhibition zones in millimeters.

\subsection{Preparation of formulation cream}

The o/w emulsion was prepared by mixing and agitating the aqueous and oil phases. For the preparation of the cream, an oily phase composed of Beeswax, Shea Butter and Apple Oil was heated up to $70^{\circ} \mathrm{C} \pm 1^{\circ} \mathrm{C}$. Aqueous phase was a solution of acetic acid $1 \%$ to which we add a precise quantity of active ingredient (Chitosan) left to dissolve for a period of time studied, we add soy lecithin as an emulsifier to stabilize the cream.

The oily phase was mixed with the aqueous phase and stirring with a thermocouple followed by a homogenizer to ensure the good homogenization of the two phases. Small drops of $\mathrm{NaOH}$ are added during the stirring to neutralize the preparation.

\subsection{Cream characterization}

The formulation cream is assessed by the determination of $\mathrm{pH}$, centrifugation test and physical analysis. Stability tests were also conducted under different storage conditions to observe the effects of different temperature ranges on the emulsions.

\subsection{Evaluation of Cream on skin}

Wistar albinos mice (20-30 g) were housed in cages $\left(20^{\circ} \mathrm{C}\right.$ to $25^{\circ} \mathrm{C}, 12$-hour light/dark cycle) and allowed free access to water and food. They were randomly divided into 2 groups $(n=3)$ as follows: group 1: was treated with Biafine; and group 2: was treated with the prepared cream. The applications will be done daily in order to compare the different scars and their evaluation

\subsection{Provocation of the burn wounds}

The animals were anesthetized. After removing the hair at the back with an electric shaver, the skin was washed with $70 \%$ surgical alcohol for disinfection. To create deep standard burn wound on the back of the mice, we trace the area to be cut and give a cylindrical shape. The metal plate was heated to a flame for 5 minutes and placed on the back of the animal for 8 seconds. In order to produce similar burns, the pressure on the skin was the same in all mice. The cream were applied instantly and repeated once daily.

\section{Results and Discussion}

\subsection{Characterization of chitosan}

The whole parameters of the prepared chitosan were tabulated in Table 1, Figure 1 showed the infrared spectrum of chitosan which allowed to determined the degree of deacetylation.

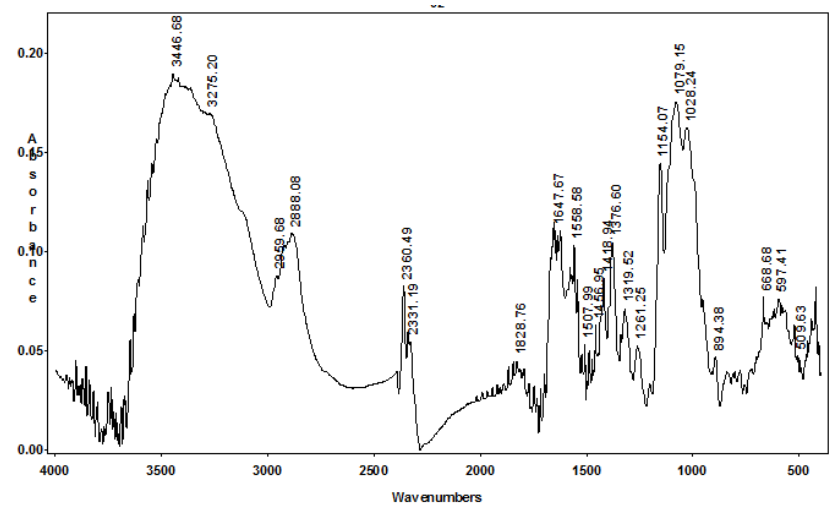

Figure 1. IR spectra of chitosan. 
Table 1. Characteristics of chitosan obtained from the Sepia bones

\begin{tabular}{cc}
\hline Characteristics & Sepia Chitosan \\
\hline Molecular weight & $65 \mathrm{KDA}$ \\
Degree of deacetylation & $70 \%$ \\
\hline
\end{tabular}

\subsection{Antibacterial mechanism of chitosan against Bacillus}

An antibacterial effect of Chitosan has been reported due to the high density of -NH3+ amino groups which gives a positive charge to the polymer, allowing the formation of poly-electrolyte complexes with negative peptidoglycans of the bacterial cell wall (Figure 2). This interaction can disrupt the cell wall and lead to inhibition of bacterial growth [13, 14].

It has also been shown that a positive particle charge is desirable to prevent particle aggregation and promote electrostatic interaction with the overall negative charge of the microbial cells [15]. Chitosan interacts with the cell membrane to alter its permeability there by impeding the entry of certain nutrients.

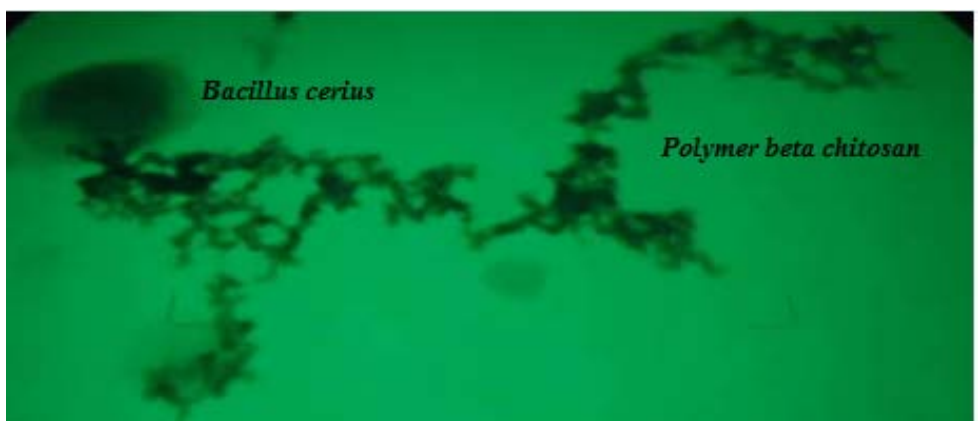

Figure 2. Beta chitosan and Bacillus cerius (interaction) observed by transmission electron microscopy (MET).

\subsection{Antimicrobial activities of chitosan}

Antimicrobial activity of chitosan against several microbial species has been recognized and is considered as one of the most important properties linked directly to their possible biological applications. In this work, the antimicrobial activity was investigated against two Gram-positive and two Gram-negative bacteria, and two fungi as shown in Table 2. The potency of chitosan against bacteria and fungi was determined through disc diffusion assay (Figure 3).

Table 2. Diameters of inhibition zones (expressed in $\mathbf{~ m m}$ ) against bacteria and fungi in the presence of chitosan after incubation $24 \mathrm{~h}$ at $37^{\circ} \mathrm{C}$ for bacteria and $72 \mathrm{~h}$ at $30^{\circ} \mathrm{C}$ for fungi

\begin{tabular}{ccc}
\hline & Diameters of inhibition zones & \\
\hline Gram - & P.aeruginosa & 52 \\
Gram + & S.aureus & 48 \\
Fungi & B.subtilis & 28 \\
& Candidas albicans & 58 \\
\hline
\end{tabular}
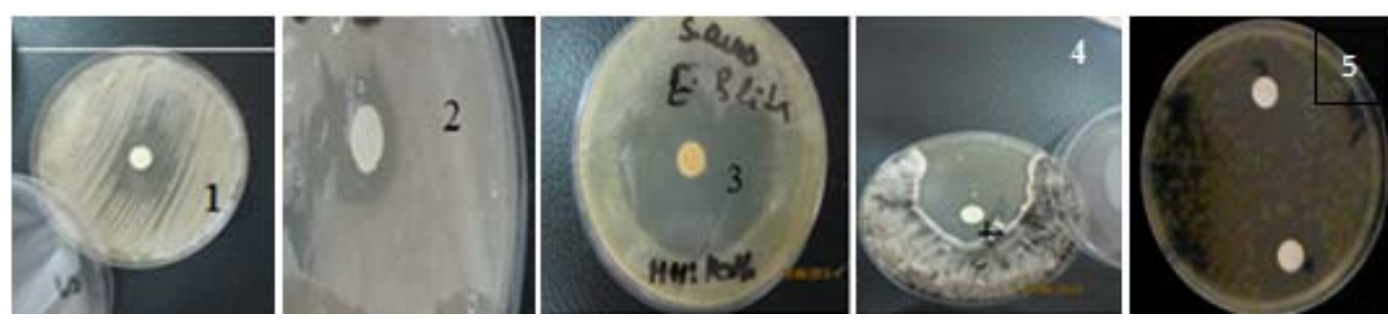

Figure 3. Disc diffusion assays showing Antimicrobial activities of chitosan against bacteria and fungi, (1) Candida albicans; (2) Pseudomonas aeroginosa; (3) Staphylococcus aureus; (4) Aspergillus niger; (5) Bacillus subtilis. 


\subsection{Cream characterization}

The formulation of chitosan is prepared and placed at $50^{\circ} \mathrm{C}$ for $24 \mathrm{~h}$.

In centrifugation test, separation of phases was not observed in the preparations after keeping them under storage for 120 days at $5^{\circ} \mathrm{C}$.

After storage under $50^{\circ} \mathrm{C}$ for 24 hours, $\mathrm{pH}$ of the formulation is 5.93

\subsection{Rheological characteristics}

The rheological test allows us to determine the viscosity of our tests as well as to make the comparison with that of the reference cream and for that we used a rheometer of mark Anton PaarModulat Compact Rheometer MCR 302.

Figure 4 showed that the prepared cream shows the same behavior as the reference cream. At low shear rate $\left(<10^{-4}\right)$, we noticed a plateau expressing a Newtonian behavior, which is translated by the fact that at such a shear rate the particles are undeformable giving a constant viscosity.

This is explained by the presence of interactions between the macromolecules of the emulsion; attractive type Van der Waals and repulsive or sometimes called the interactions of elastic return and that does not register any change on the structural level and therefore the viscosity remains constant.

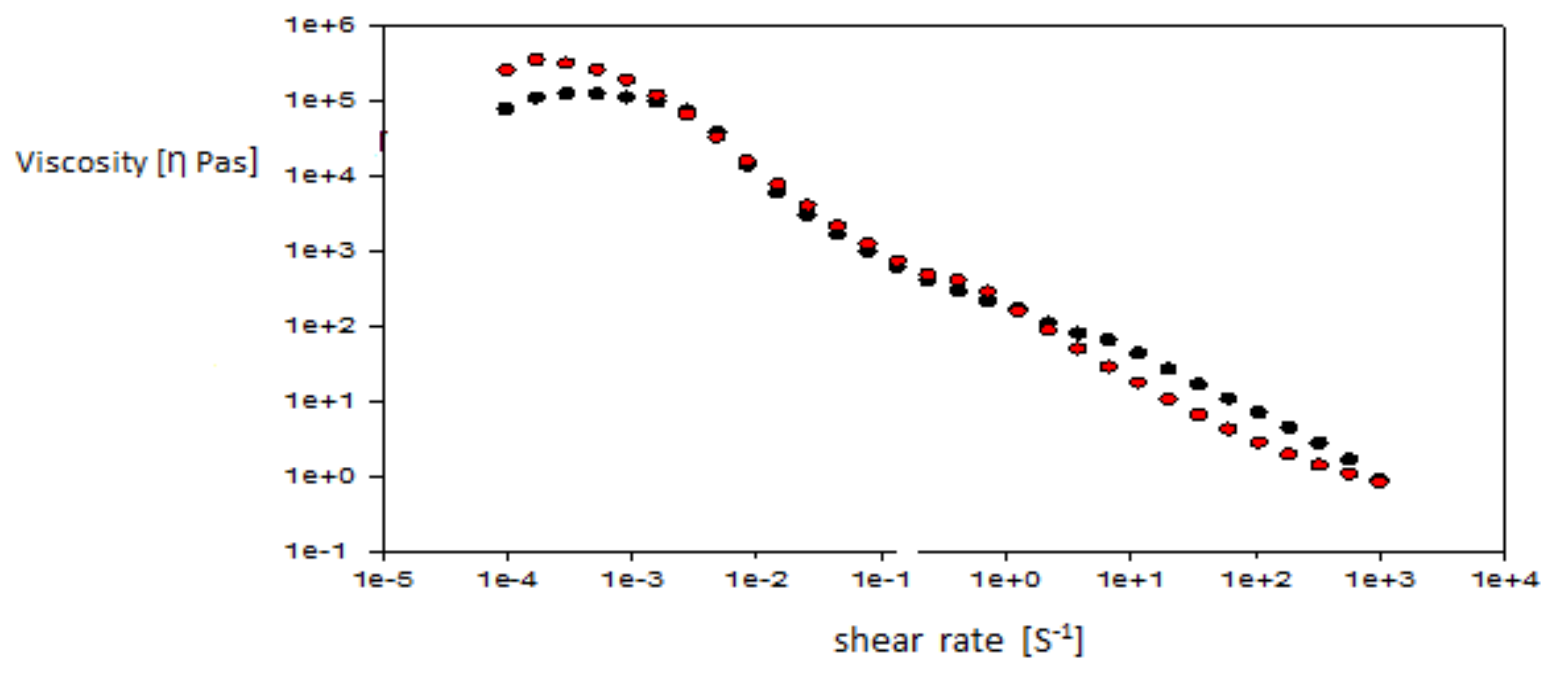

\section{- Referencecream BIAFINE}

Figure 4. Apparent viscosity versus shear rate for prepared cream with chitosan and reference cream.

For a shear rate above $10^{-3} \mathrm{~s}^{-1}$, the viscosity drops sharply expressing a non-Newtonian behavior (rheofluidizing). Thus, the flow curve corresponds to two times of the application of the cream, the first has the taking of the cream from the pot and the second to the spreading of the latter. This is explained by the fact that the repulsive forces are high compared to the attractive forces, so we will have a destructuring of the emulsion and the viscosity tends to decrease.

It should be noted that the rheological behavior of cream formulated in the laboratory is similar to that of reference creams. The values of apparent viscosity were determined from mathematical models.

\subsection{Sensorial analysis}

The sensory analysis is a very important parameter directly related to the affinity of each consumer.

In order for this analysis to have more value, it is necessary to reproduce it on the maximum number of people possible. Thus, the sensory analysis consists in studying in an orderly and structured way the properties of a product in order to be able to describe it, to classify it or to improve it in an extremely objective and rigorous way.

For that formulation was tested by 20 people, an average of the notation attributed for each criterion (the aspect, the richness, the diffusion, the spreading, the unctuousness, the filmogenic effect). The results are grouped on Table 3. 
Table 3. Average of the criteria of the sensory analysis of cream

\begin{tabular}{cccccc}
\hline Spreading & Appearance & Richness & Ouctuosity & Diffusion & Film \\
\hline 3.8 & 3.975 & 3.725 & 3.2 & 3.65 & 3.2 \\
\hline
\end{tabular}

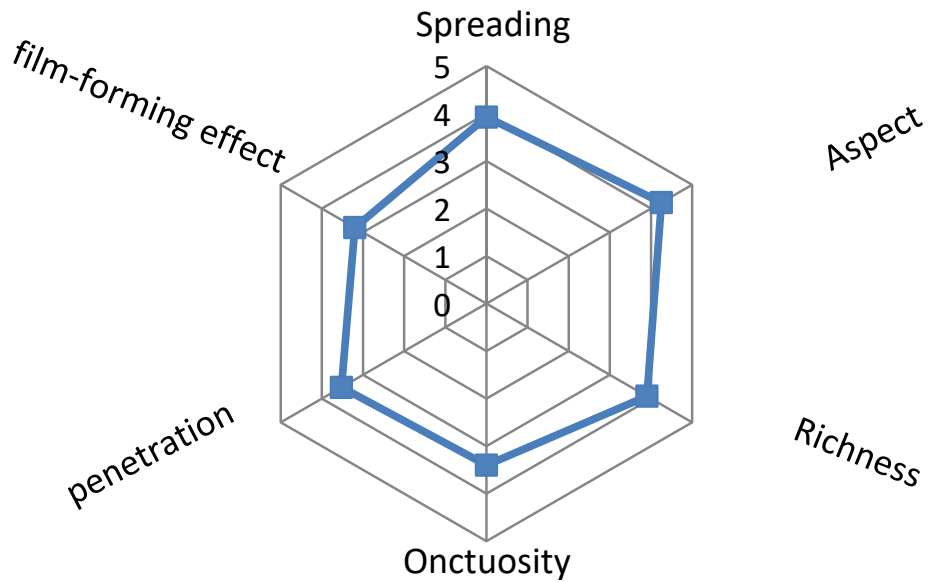

Figure 5. Sensory analysis diagram.

According to the diagram obtained by the sensory analysis (Figure 5), our cream was appreciated by the 20 people.

\subsection{In vivo experimental study}

\subsubsection{Morphological evaluation}

Figure 6 demonstrated the representative sets of wounds photographs of mice. On the third day, the burns showed a brown color due to the formation of the scab. After 4 days, the areas of the wounds tended to decrease, and it is only after the 7th day that significant decreases are observed. For the reference cream it by the 21th day, that the wounds are healed.

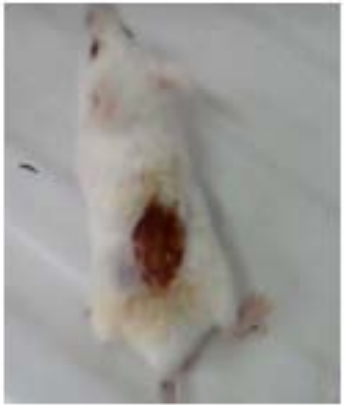

(a)

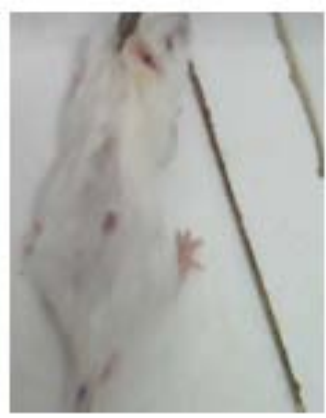

(b)

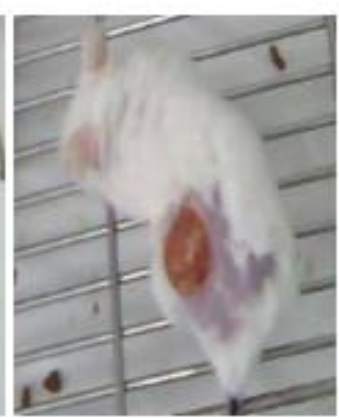

(c)

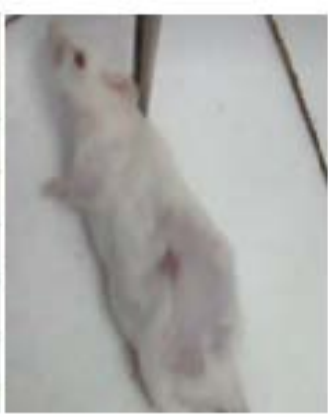

(d)

Figure 6. Representative photographs of macroscopic appearance of wounds excised on mice. (a) day 1 group treated with chitosan cream; (b) day 7 group treated with chitosan cream; (c) day 1 group treated with reference cream; (d) day 21 group treated with reference cream.

\section{Conclusion}

The galenic formulation has enabled the development of an antimicrobial and healing cream based on a biopolymer, beta chitosan as active principle, shea butter, sweet almond oil and beeswax as excipients. Animals with skin infections (deep wounds and burns) were treated with the cream and all recovered successfully. These preclinical tests confirmed the in vitro antimicrobial activities of the active principle. The prepared cream has thus proven to be effective and can be sold at a lower cost compared to modern antimicrobials and healing agents and will therefore be accessible to all. 


\section{References}

[1] Cheung, R. C. F., et al. (2015). Chitosan: an update on potential biomedical and pharmaceutical applications. Marine Drugs, 2015, 13(8): pp. 5156-5186.

[2] Arrouz, F., et al. (2017). Chitin and chitosan: study of the possibilities of their production by valorization of the waste of crustaceans and cephalopods rejected in essaouira. Journal of Materials and Environmental Sciences, 2017, 8(7): pp. $2251-2258$.

[3] Tokathi, K. and Demirdoven. Optimization of chitin and chitosan production from shrimp wastes and characterization. Journal Processing and Preservation. DOI: 10.111/jfpp.13494.

[4] Soulié, M. C., et al. (2006). Botrytis cinerea virulence is drastically reduced after disruption of chitin synthase class III gene (Bcchs3a). Cellular Microbiology, 2006, 8(8): pp. 1310-1321.

[5] Rabea, E. I., et al. (2003). Chitosan as antimicrobial agent: applications and mode of action. Biomacromolecules, 2003, 4(6): pp. 1457-1465.

[6] Şenel, S. and S. J. McClure. (2004). Potential applications of chitosan in veterinary medicine. Advanced Drug Delivery Reviews, 2004, 56(10): pp. 1467-1480.

[7] Juneja, V. K., et al. (2006). Chitosan protects cooked ground beef and turkey against Clostridium perfringens spores during chilling. Journal of Food Science, 2006, 71(6): pp. M236-M240.

[8] Klong Thong, et al. (2020). Chitisan biomedical applications for the treatment of viral disease: Adota mining model using bibliometric predictive intelligence. Journal of Chemistry, 2020. ID 6612034, p. 12.

[9] Nor Janah, M. S. and Khairul, A. M. A. (2017). Composite film of chitosan loaded norfloxacin with improved flexibity and antibacterial activity for wound dressing application. Oriental Journal of Chemistry, 2017, 33(2): pp. 628-636.

[10] Cuero, R. G. (1999). Antimicrobial action of exogenous chitosan. Exs, 1999, 87: pp. 315-333.

[11] Delorino, M. and S. Cresidio. (2009). Investigation of chitosan from squid pen as Scar Remover. World Appl. Sci. J., 2009, 5: pp. 98-103.

[12] Khan, T. A., K. K. Peh, and H. S. Ch'ng. (2002). Reporting degree of deacetylation values of chitosan: the influence of analytical methods. J Pharm Pharmaceut Sci., 2002, 5(3): pp. 205-212.

[13] Krajewska, B., P. Wydro, and A. Jańczyk. (2011). Probing the modes of antibacterial activity of chitosan. Effects of pH and molecular weight on chitosan interactions with membrane lipids in Langmuir films. Biomacromolecules, 2011, 12(11): pp. 4144-4152.

[14] Cruz-Romero, M., et al. (2013). Antimicrobial activity of chitosan, organic acids and nano-sized solubilisates for potential use in smart antimicrobially-active packaging for potential food applications. Food Control, 2013, 34(2): pp. 393-397.

[15] Qi, L., et al. (2004). Preparation and antibacterial activity of chitosan nanoparticles. Carbohydrate Research, 2004, 339(16): pp. 2693-2700. 